\title{
The Impact of Tumor Volume on Outcomes after Radical Prostatectomy: Implications for Prostate Cancer Screening
}

\author{
James D. Brooks ${ }^{1, *}$, Robert Tibshirani ${ }^{2}$, Michelle Ferrari ${ }^{1}$, Joseph C. Presti, Jr. ${ }^{1}$, Harcharan Gill ${ }^{1}$ and \\ Christopher R. King ${ }^{3}$
}

${ }^{1}$ Departments of Urology, ${ }^{2}$ Health Research and Policy and Statistics and ${ }^{3}$ Radiation Oncology, Stanford University,
Stanford, California, USA

\begin{abstract}
Purpose: For screening to make an impact on prostate cancer mortality, detection of potentially lethal cancers at an early stage when they are low volume should result in improved recurrence and death rates after treatment.

Patients and Methods: The effect of tumor volume on prostate cancer recurrence and death was evaluated in 764 men who underwent radical prostatectomy between 1984 and 2004, with particular attention focused on patients with moderate and high risk features.

Results: Tumor volume was a powerful predictor of recurrence in men after radical prostatectomy with moderate and high risk features, even after accounting for the effects of percentage Gleason pattern 4/5 cancer, extracapsular extension, seminal vesicle invasion, lymph node metastasis, pre-operative PSA, and surgical margin involvement. In a subset of 159 patients for whom pre-operative PSA velocity was available, tumor volume predicted recurrence in those in the highest risk category (PSAV $>2 \mathrm{ng} / \mathrm{ml} / \mathrm{yr}$ ). Tumor volume, along with percent grade $4 / 5$ and positive surgical margins, was significantly associated with prostate cancer specific death.

Conclusions: The association of volume with outcome after radical prostatectomy, particularly in high risk patients, suggests that screening has made a positive impact on prostate cancer mortality. Future screening efforts should be directed at finding cancers with moderate and high risk features at low volume.
\end{abstract}

Key Words: Prostate cancer, tumor volume, gleason grade, prostate specific antigen velocity, death, recurrence.

\section{INTRODUCTION}

Over the past 10-15 years, wide application of PSA screening has resulted in significant prostate cancer stage migration and decreased size and stage of incident cancers in radical prostatectomy series [1]. Since 1992, the number of prostate cancer deaths has also decreased significantly. However, in the absence of randomized trials, it is unknown whether screening can be credited with the decline in prostate cancer mortality rates. Prostate cancer death rates have dropped in parallel with those of lung and colon cancers, and it is possible that other factors, such as decreases in the incidence of smoking or changes in diet, account for most or all of these improvements.

While the Prostate, Lung, Colorectal and Ovarian (PLCO) Cancer Screening Trial and European Randomized Study of Screening for Prostate Cancer (ERSPC) trials address directly whether prostate cancer screening can reduce deaths, recent studies have raised questions whether screening programs can impact cancer death rates [2,3]. Lung cancer screening by spiral CT scans of the chest does increase

\footnotetext{
*Address correspondence to this author at the Department of Urology, Room S287, Stanford University Medical Center, 300 Pasteur Drive, Stanford, CA 94305-5118, USA; Tel: 650-725-5546; Fax: 650-723-4200; E-mail: jdbrooks@stanford.edu

Supported by the National Institutes of Health grant CA111782 and a Stanford University Bio-X Interdisciplinary Initiatives Award
}

detection of low volume, stage 1 cancers and the number of surgeries performed [4]. However, screening is not associated with a decrease in the absolute numbers of lung cancer deaths, presumably because the potentially lethal cancers have already metastasized by the time they are discovered [5]. Furthermore, lung cancer screening appears to detect a subset of "incidental" lung cancers that will never kill the patient and do not require treatment. Therefore, detection of high risk lung cancers at a low stage and volume might not be effective at reducing death, but does increase the number of apparently unnecessary surgeries. Could the same be true of prostate cancer?

The clinical features of high risk and potentially lethal prostate cancers are well understood and include high Gleason grade, markedly elevated pre-treatment serum PSA levels, advanced stage and high tumor volume. Recently, several studies have refined the definition of high risk prostate cancers. The percentage of Gleason's grade pattern 4 and 5 has been demonstrated to outperform simple expressions of the Gleason sum in predicting treatment outcome [6]. In addition, several large series have shown that pre-treatment PSA velocity identifies patients at high risk for treatment failure and death after local therapy while absolute PSA level does not [7-9]. Since prostate cancer screening has been associated with significant declines in tumor volume, the question arises whether smaller volumes in high risk cancers are associated with better outcomes [1]. Stated an- 
other way, are there lethal forms of prostate cancer for which tumor volume will not influence outcome and screening will not be effective? To evaluate whether tumor volume influences outcome, we analyzed its relationship to recurrence and prostate cancer death after radical prostatectomy, focusing on patients with high-risk features.

\section{PATIENTS AND METHODS}

\section{Patient Population}

Included in this study were 789 patients who had signed an informed consent approved by our Institutional Review Board that allowed collection clinical and pathological data. All patients had undergone radical retropubic prostatectomy for clinically localized prostate cancer between 1984 and 2004. Patients were excluded who had undergone transurethral resection of the prostate pre-operatively (8) or had been treated with neoadjuvant hormonal therapy (17). All patients were followed at approximately 6 month intervals with serum PSA determinations. Biochemical failure was defined by a serum PSA $>0.07 \mathrm{ng} / \mathrm{ml}$ and rising on subsequent de- terminations. Review of death certificates, direct contact of the patients' families, or review of the medical record confirmed prostate cancer specific death in 24 patients. Preoperative PSA velocity (PSAV) was calculated using a simple linear regression on two or more values obtained prior to diagnosis as described previously [9]. Of the 764 patients, 159 had 2 or more pre-operative PSA values for calculation of PSAV. Patient characteristics are summarized in Table $\mathbf{1}$. Values that appeared to be normally distributed were reported as means \pm standard deviation (SD), and those with a skewed distribution were reported as medians with interquartile ranges.

\section{Pathological Evaluation}

Radical prostatectomy specimens were fixed for 24 hours by perfusion with $10 \%$ formalin, and processed at $3 \mathrm{~mm}$ intervals as described previously [6]. Tumor volume was determined by computer planimetry, a method that has been demonstrated to provide the most accurate assessment of tumor volume [10]. Tumor volume was measured on the

Table 1. Patient Characteristics

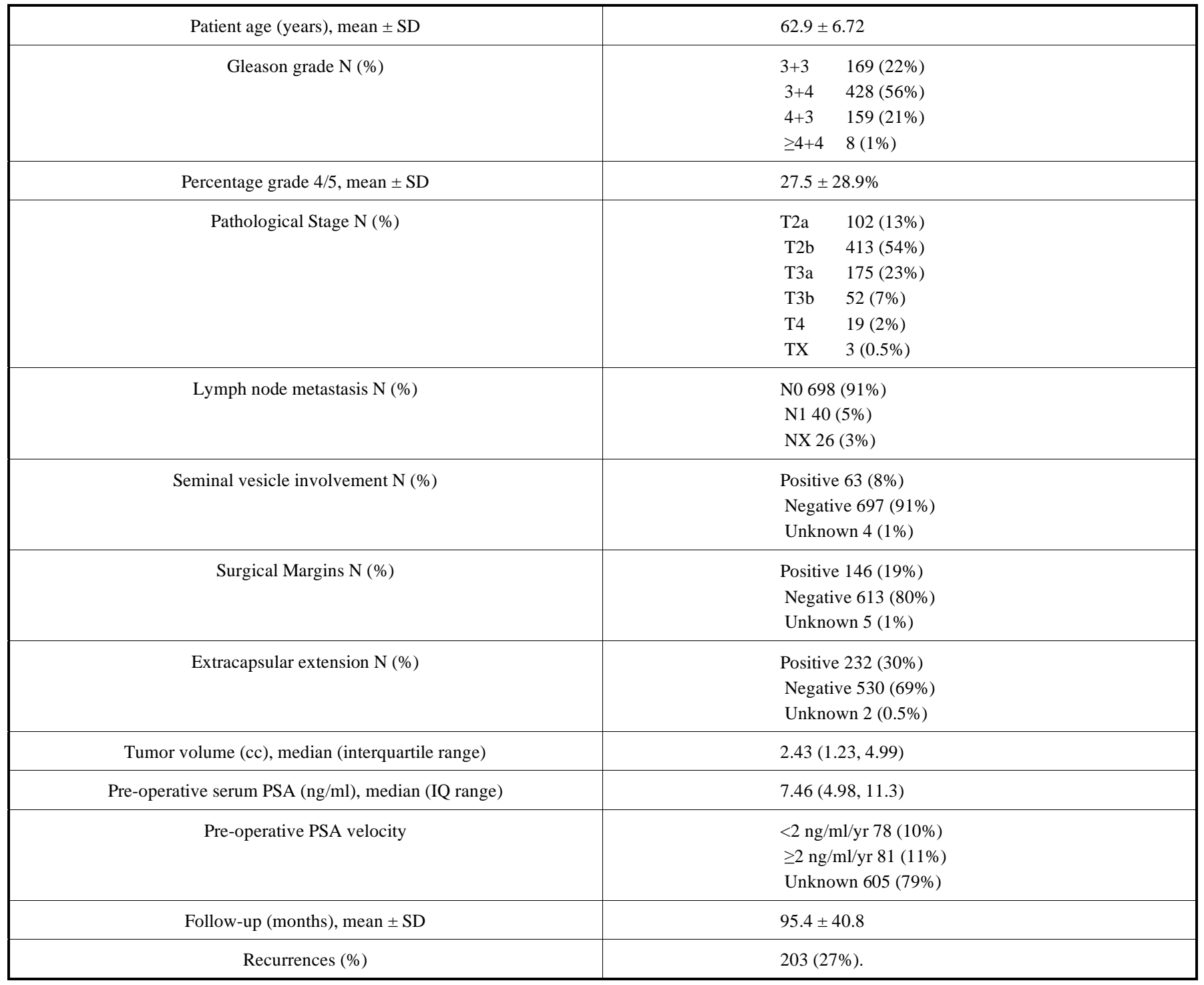


largest incident tumor only. Tumor volume did not include secondary cancers and there were no cases of secondary cancers with grades that exceeded the largest incident cancer. Surgical margin status, pathological staging, Gleason scoring and estimation of percentage of Gleason pattern 4/5 were performed by a single pathologist as described in detail elsewhere [1]. Estimation of percentage of Gleason pattern $4 / 5$ was not performed on 2 patients and tumor volume was not available on 18 patients.

\section{Statistical Analysis}

Associations between clinical and pathological variables were tested by Pearson correlation. Univariate and multivariate Cox proportional hazards models were used to evaluate the relationships between the clinical and pathological variables and disease recurrence or prostate cancer death. Survival analysis was performed using the Kaplan Meier and log rank tests. Statistical tests were all two-sided. All statistical analyses were carried out in $\mathrm{R}$ package, version 2.4.1.

\section{RESULTS}

To understand the importance of volume on outcome after radical prostatectomy, we tested its effects on recurrence and prostate cancer specific death in the context of other known determinants of outcome including percentage Gleason grade $4 / 5$, presence of extracapsular extension, surgical margin status, lymph node involvement, seminal vesicle involvement, pre-operative serum PSA levels and preoperative PSA velocity. Since traditional Gleason grading (or Gleason sum) is not independent of percentage 4/5 and shows poorer performance in predicting outcome, we did not include it in this analysis [6]. Likewise, we did not include pathological stage since we analyzed independently the variables encompassed in staging (seminal vesicle invasion, lymph node metastasis, extracapsular extension). Table 2 shows a correlation matrix of the clinical and pathological parameters. Although there was only modest correlation between any two variables, it is notable that the correlation between tumor volume and pre-operative serum PSA levels was the greatest. Regardless, the small to modest correlations between parameters means that associations of any single variable with outcome should be interpreted with caution, particularly at borderline $p$ values.

In univariate analysis, all seven clinical and pathological variables were highly associated with time to recurrence after radical prostatectomy, as expected (all $p<0.0001$, Table 3). When all variables were included in the model, volume, percentage Gleason grade 4/5, positive surgical margins,

Table 2. Pearson Correlation Between Clinical and Pathological Parameters

\begin{tabular}{|c|c|c|c|c|c|c|}
\hline & $\begin{array}{c}\text { Tumor } \\
\text { Volume }\end{array}$ & Grade 4/5 & PSA & $\begin{array}{c}\text { Seminal Vesicle } \\
\text { Involvement }\end{array}$ & $\begin{array}{c}\text { Lymph Node } \\
\text { Involvement }\end{array}$ & $\begin{array}{c}\text { Extracapsular } \\
\text { Extension }\end{array}$ \\
\hline \hline Tumor volume & 1.000 & & & & & \\
\hline Grade 4/5 & 0.276 & 1.000 & & & & \\
\hline PSA & 0.489 & 0.282 & 1.000 & & & \\
\hline Seminal Vesicle involvement & 0.399 & 0.326 & 0.382 & 1.000 & & \\
\hline Lymph node involvement & 0.315 & 0.224 & 0.368 & 0.369 & 1.000 & \\
\hline Extracapsular Extension & 0.344 & 0.307 & 0.258 & 0.345 & 0.251 & 1.000 \\
\hline Surgical Margins & 0.345 & 0.206 & 0.264 & 0.235 & 0.155 & 0.343 \\
\hline
\end{tabular}

All correlation coefficients highly significant at $\mathrm{p}<0.0001$.

Table 3. Univariate Analysis of 7 Clinical and Pathological Variables and Time to Recurrence

\begin{tabular}{|c|c|c|c|c|c|c|}
\hline Feature & Coefficient & SE & $z$ Score & $P$ value & HR & $95 \% \mathrm{CI}$ \\
\hline Tumor volume* & 0.065 & 0.0055 & 11.8 & $<0.0001$ & 1.07 & 1.06 to 1.08 \\
\hline Sem Ves Invas & 2.05 & 0.16 & 12.8 & $<0.0001$ & 7.77 & 5.67 to 10.6 \\
\hline Extracaps Ext & 1.78 & 0.15 & 11.9 & $<0.0001$ & 5.91 & 4.42 to 7.92 \\
\hline PSA $\neq$ & 0.037 & 0.0035 & 11.0 & $<0.0001$ & 1.04 & 1.03 to 1.05 \\
\hline Positive LN & 2.01 & 0.19 & 10.7 & $<0.0001$ & 7.46 & 5.15 to 10.8 \\
\hline
\end{tabular}

*HR for each $1 \mathrm{cc}$ increase in tumor volume.

†HR for each $1 \%$ increase in grade $4 / 5$ cancer

†HR for each $1 \mathrm{ng} / \mathrm{ml}$ increase in serum PSA. 
Table 4. Cox Regression Analysis of 7 Clinical and Pathological Variables and Time to Recurrence

\begin{tabular}{|c|c|c|c|c|c|c|}
\hline Feature & Coefficient & SE & $z$ Score & $P$ Value & HR & $95 \% \mathrm{CI}$ \\
\hline Tumor volume* & 0.025 & 0.0088 & 2.874 & 0.0004 & 1.03 & 1.01 to 1.04 \\
\hline Sem Ves Invas & 0.376 & 0.209 & 1.802 & 0.07 & 1.46 & 0.97 to 2.19 \\
\hline Extracaps Ext & 1.186 & 0.174 & 6.824 & $<0.0001$ & 3.27 & 2.33 to 4.60 \\
\hline PSA & -0.00065 & 0.0055 & -0.117 & 0.91 & 1.00 & 0.99 to 1.01 \\
\hline Positive LN & 0.916 & 0.252 & 3.635 & $<0.0001$ & 2.50 & 1.52 to 4.09 \\
\hline
\end{tabular}

*HR for each $1 \mathrm{cc}$ increase in tumor volume.

†HR for each $1 \%$ increase in grade $4 / 5$ cancer.

†HR for each $1 \mathrm{ng} / \mathrm{ml}$ increase in serum PSA.

extracapsular extension and lymph node involvement were associated with time to recurrence, while seminal vesicle invasion and pre-operative PSA levels were not (Table 4). In step-wise regression, only pre-operative PSA did not contribute to the model. Compensation for the skewed distribution of PSA and volume by log transformation did not appreciably affect performance of the models or the variables included.

To better understand the effects of volume on recurrence after radical prostatectomy, we divided the cohort into those with little Gleason grade $4 / 5$ cancer $(<5 \%)$, moderate amounts $(5-49 \%)$ and high amounts $(\geq 50 \%)$. These values were selected based on an analysis of a subset of these patients showing highly favorable outcomes for all patients with little grade 4/5 cancer, and high recurrence rates that remained constant in patients after the percentage of grade 4/5 cancer exceeded 50\% [6]. As seen in Fig. 1, volume was highly predictive of outcome in high risk (grade $4 / 5 \geq 50 \%$, $p<0.0001, \log$ rank test) and intermediate risk tumors (grade $4 / 55-49 \%, p<0.0001$, log rank test). Volume did not predict recurrence in low grade cancers ( $p=0.08, \log$ rank test), possibly due to the low rate of recurrence in these patients. A similar lack of association between volume and recurrence for low grade cancers has been reported recently [11].

Several studies have found PSAV $>2 \mathrm{ng} / \mathrm{ml} / \mathrm{yr}$ as a critical cut-off for defining patients at high risk for recurrence after local therapy or death from prostate cancer [7-9]. We were able to calculate pre-operative PSAV on a subset of 159 patients in our study. This subset matched the rest of the study population in the distribution of clinical and pathological parameters except that no patients with positive lymph nodes were included. As we reported previously, PSAV predicted time to recurrence, both as a continuous and categorical (i.e. using a $2 \mathrm{ng} / \mathrm{ml} / \mathrm{yr}$ cut-off) variable [9]. In multivariate analysis, PSAV remained a significant predictor of time to failure as did percentage grade $4 / 5$ and the presence of extracapsular extension. After exclusion of PSA, extracapsular extension, surgical margins, and seminal vesicle invasion from the model, tumor volume $(p<0.01)$, percentage grade $4 / 5(p<0.001)$ and PSAV $>2(p<0.001)$ all predicted failure. Interestingly, for patients with PSAV $>2$, tumor volume was highly predictive of outcome ( $p<0.05, \log$ rank test), while for those with PSAV $<2$, volume was not $(p=0.5)$ (Fig. 2). Therefore, for high risk individuals, defined by PSAV $>2$, high tumor volume again was predictive of an adverse outcome, while in lower risk individuals, it was not.

In the full cohort of 764 patients, 24 died of prostate cancer after radical prostatectomy (median time to death 105 mos., range 28 - 202 mos.). Tumor volume, percentage grade $4 / 5$, seminal vesicle invasion, extracapsular extension, lymph node metastasis, positive surgical margins and preoperative PSA were all associated with time to death in univariate analysis (Table 5). In multivariate analysis, only volume $(p=0.0004)$, percentage grade $4 / 5(p=0.01)$ and positive surgical margins $(p=0.01)$ were associated with death (Table 6). Again, log transformation of tumor volume and PSA did not change the results appreciably. Step-wise regression confirmed that only tumor volume $(p<0.001)$, percentage grade $4 / 5(p<0.001)$ and positive surgical margins $(p=0.003)$ were significantly associated with death. Furthermore, Kaplan-Meier analysis of the patients with moderate and high risk cancers (grade $4 / 5>5 \%$ ) showed that increasing volume was highly predictive of death due to prostate cancer after radical prostatectomy $(p=0.0003$, Log rank test) (Fig. 3).

\section{DISCUSSION}

Cancer volume is a powerful predictor of outcome after radical prostatectomy, particularly in men with moderate and high risk prostate cancers. In men with high risk features, defined by Gleason grade $4 / 5>50 \%$ or preoperative PSAV $>2 \mathrm{ng} / \mathrm{ml} /$ year, tumor volume showed a highly significant association with time to treatment failure. Furthermore, moderate to high risk prostate cancers (grade $4 / 5>5 \%$ or Gleason grade 7 and above) showed a strong association between increased tumor volume and prostate cancer specific death. These findings imply that screening procedures that detect moderate and high-risk cancers at lower volumes will decrease recurrences after surgery and improve prostate cancer death rates. Therefore, the decrease in incident tumor volume over the past 2 decades strongly suggests that screening has played a significant role in the decrease in prostate cancer deaths [1]. 
Gleason Grade 3+3

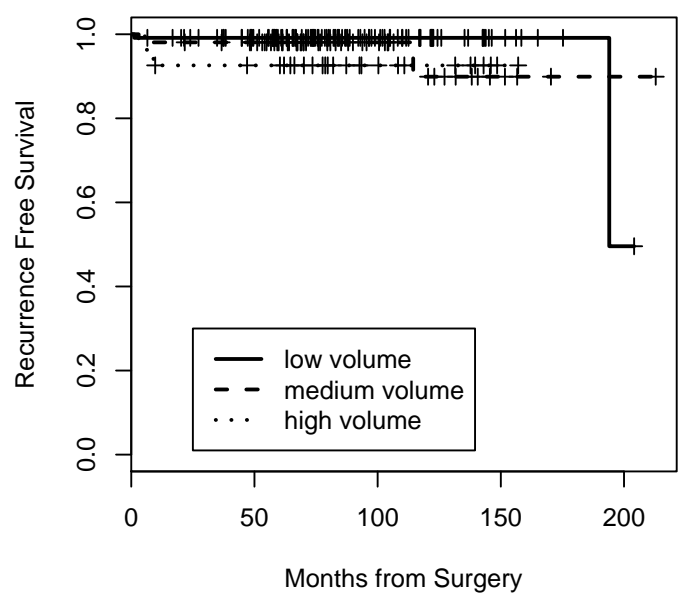

4+3 and Above

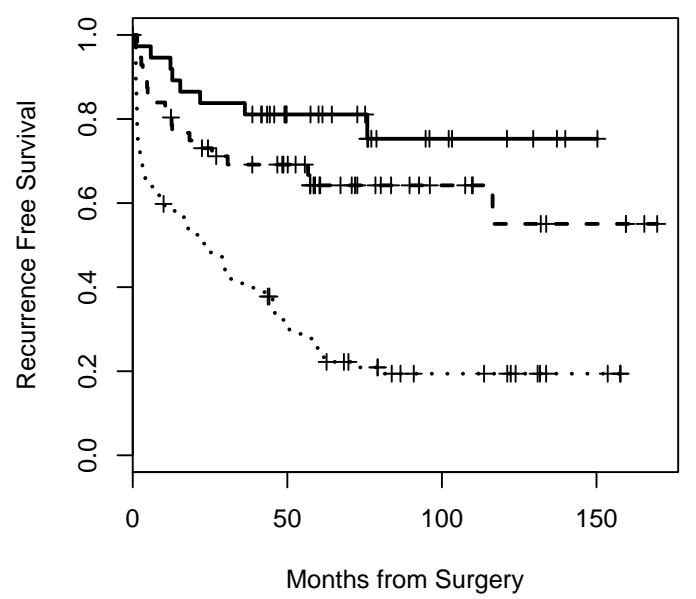

Gleason Grade 3+4

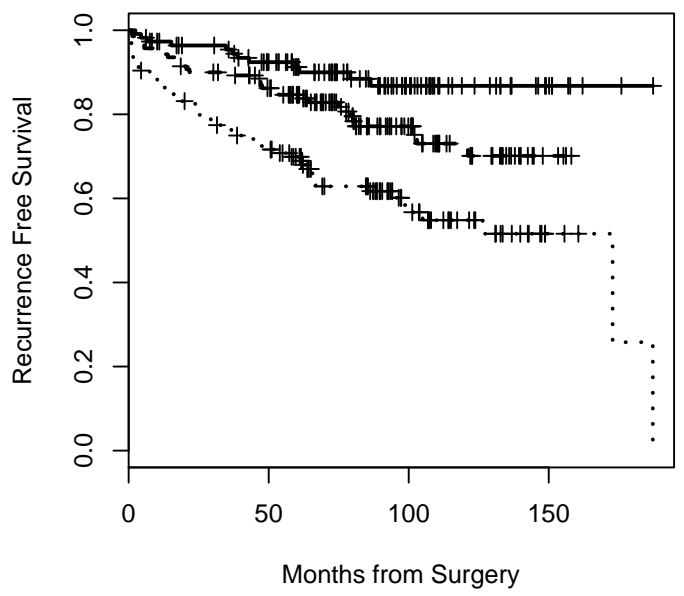

Fig. (1). Kaplan-Meier analysis representing time to recurrence after surgery for tumor volume stratified by Gleason grade. Gleason grade was divided into prognostically relevant groups as defined in the text. Tumor volume was divided into tertiles of low ( $<1.65 \mathrm{cc})$, medium $(1.65-4.15 \mathrm{cc})$ and high $(>4.15 \mathrm{cc})$ volume disease. Each tick mark represents a censored value. Volume was highly prognostic for high grade ( $p<0.0001, \log$ rank test) and intermediate grade $(p<0.0001, \log$ rank test), but not for low grade prostate cancers $(p=0.08, \log$ rank test $)$.

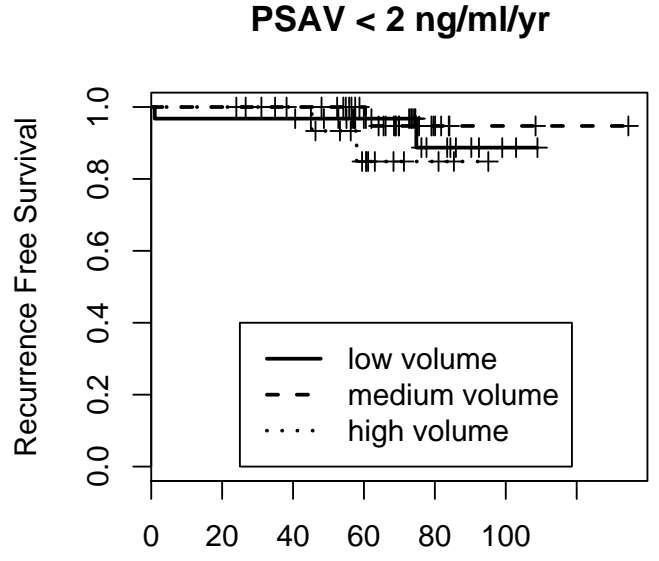

Months from Surgery
PSAV > $2 \mathrm{ng} / \mathrm{ml} / \mathrm{yr}$

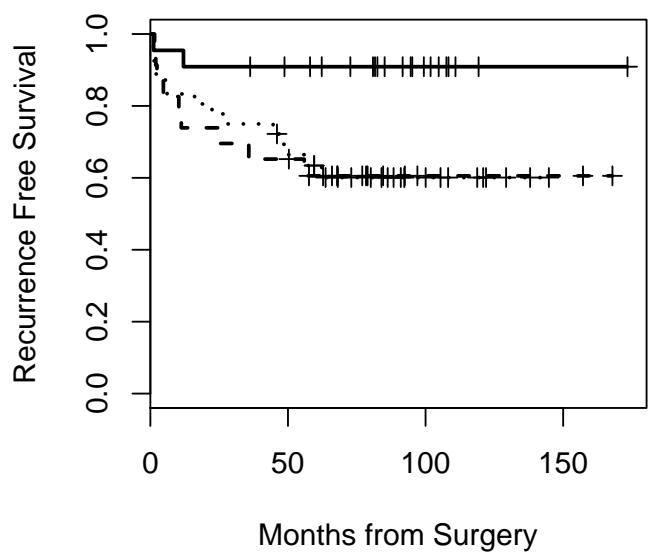

Fig. (2). Kaplan-Meier analysis representing time to recurrence after surgery for tumor volume stratified by pre-operative PSA velocity (PSAV). A cut-off value of PSAV of $2 \mathrm{ng} / \mathrm{ml} / \mathrm{yr}$ was selected since it has been previously defined as highly prognostic for prostate cancer recurrence and prostate cancer specific death (see text). Tumor volume was divided into tertiles as in Fig. (1) and each tick mark represents a censored value. Tumor volume was predictive of time to recurrence for patients with pre-operative PSAV $>2(p<0.05$, log rank test), but not for those with low PSAV $<2(p=0.5)$. 
Table 5. Univariate Analysis of 7 Clinical and Pathological Variables and Time to Death from Prostate Cancer

\begin{tabular}{|c|c|c|c|c|c|c|}
\hline Tumor volume* & 0.085 & 0.014 & 5.69 & $<0.0001$ & 1.09 & 1.06 to 1.12 \\
\hline Sem Ves Invas & 1.64 & 0.42 & 3.9 & $<0.0001$ & 5.15 & 2.26 to 11.7 \\
\hline PSA & 0.026 & 0.0095 & 2.73 & 0.006 & 1.03 & 1.01 to 1.05 \\
\hline Positive LN & 1.84 & 0.46 & 4.0 & $<0.0001$ & 6.30 & 2.55 to 15.5 \\
\hline
\end{tabular}

*HR for each $1 \mathrm{cc}$ increase in tumor volume.

$\dagger$ HR for each $1 \%$ increase in grade $4 / 5$ cancer.

\$HR for each $1 \mathrm{ng} / \mathrm{ml}$ increase in serum PSA.

Table 6. Cox Regression Analysis of 7 Clinical and Pathological Variables and Time to Death from Prostate Cancer

\begin{tabular}{|c|c|c|c|c|c|c|}
\hline Feature & Coefficient & SE & $z$ Score & P Value & HR \\
\hline \hline Tumor volume* & 0.064 & 0.0179 & 3.554 & $\mathbf{0 . 0 0 0 4}$ & 1.07 & 1.03 to 1.10 \\
\hline \% Grade 4/5† & 0.020 & 0.0079 & 2.573 & $\mathbf{0 . 0 1}$ & 1.02 & 1.01 to 1.04 \\
\hline Sem Ves Invas & 0.400 & 0.533 & 0.751 & 0.45 & 0.52 to 4.24 \\
\hline Extracaps Ext & 0.188 & 0.604 & 0.311 & 0.76 & 1.21 & 0.37 to 3.94 \\
\hline Positive margins & 1.332 & 0.519 & 2.567 & $\mathbf{0 . 0 1}$ & 3.79 & 1.37 to 10.5 \\
\hline PSA & -0.025 & 0.014 & -1.723 & 0.09 & 0.97 & 0.95 to 1.00 \\
\hline Positive LN & 0.904 & 0.584 & 1.549 & 0.12 & 2.47 \\
\hline
\end{tabular}

*HR for each $1 \mathrm{cc}$ increase in tumor volume.

$\dagger$ HR for each $1 \%$ increase in grade $4 / 5$ cancer.

\$HR for each $1 \mathrm{ng} / \mathrm{ml}$ increase in serum PSA.

Unfortunately, our data also suggest that detection of high-risk cancers when they are low volume will not eliminate prostate cancer deaths. Some patients with relatively low tumor volumes still suffer recurrences and death after treatment. For example, 3 of 310 patients with tumor volumes less than $2 \mathrm{cc}$ eventually died of prostate cancer, including an individual with a preoperative PSA of $4.54 \mathrm{ng} / \mathrm{ml}$, a tumor volume of $0.29 \mathrm{cc}$, and only $40 \%$ grade $4 / 5$. These observations suggest that there might be a small subset of cancers that are highly aggressive early in their course and not curable by local therapy, even when they are discovered at a relatively small volume. It is also possible that there are host, environmental (e.g. immune function, diet) or stochastic factors that contribute to the progression of some cancers. These small lethal tumors could not be identified by clinical measures of aggressiveness currently in use, such as grade, stage or PSA level. New approaches will need to be developed to better define, detect and treat this subset of cancers.

Since tumor volume is a powerful predictor of outcome, it is important to consider how potentially aggressive tumors can be discovered at a low enough volume to be curable. Over the course of this study, most cancers were detected due to an abnormal rectal examination or serum PSA $>4$ $\mathrm{ng} / \mathrm{ml}$, and mean tumor volume decreased from $5.2 \mathrm{cc}$ to 2.1 cc. However, it is unknown whether continued application of this screening strategy or modified strategies will result in further volume decreases in high risk cancers. For instance, it is well known that high-grade cancers often produce less PSA per volume of cancer tissue, meaning that they are discovered at a relatively high volume when treatments are less effective. In the Prostate Cancer Prevention Trial, 67 of 449 (14.9\%) cancers diagnosed at the end of study biopsy showed moderate and high-risk features despite normal PSA levels and rectal examinations [12]. Although more patients would have been captured if a PSA level of $2.5 \mathrm{ng} / \mathrm{ml}$ rather than $4.0 \mathrm{ng} / \mathrm{ml}$ were used, a significant number of moderate and high risk tumors would have gone undetected, allowing these tumors to grow and worsening chances for a favorable outcome. More questions surround the use of PSA velocity for identifying patients with high risk disease. Longitudinal studies have shown that PSA rises as little as $0.35 \mathrm{ng} / \mathrm{ml} / \mathrm{yr}$ can be associated with prostate cancer death [13], yet practical application of such PSAV cut-offs is confounded by the significant overlaps in PSAV for future localized (and potentially clinically indolent) prostate cancers and benign prostate diseases, especially considering the inherent noise in PSA determinations. In addition, it is unknown whether ap- 
Death Gleason Grade 3+4 and Above

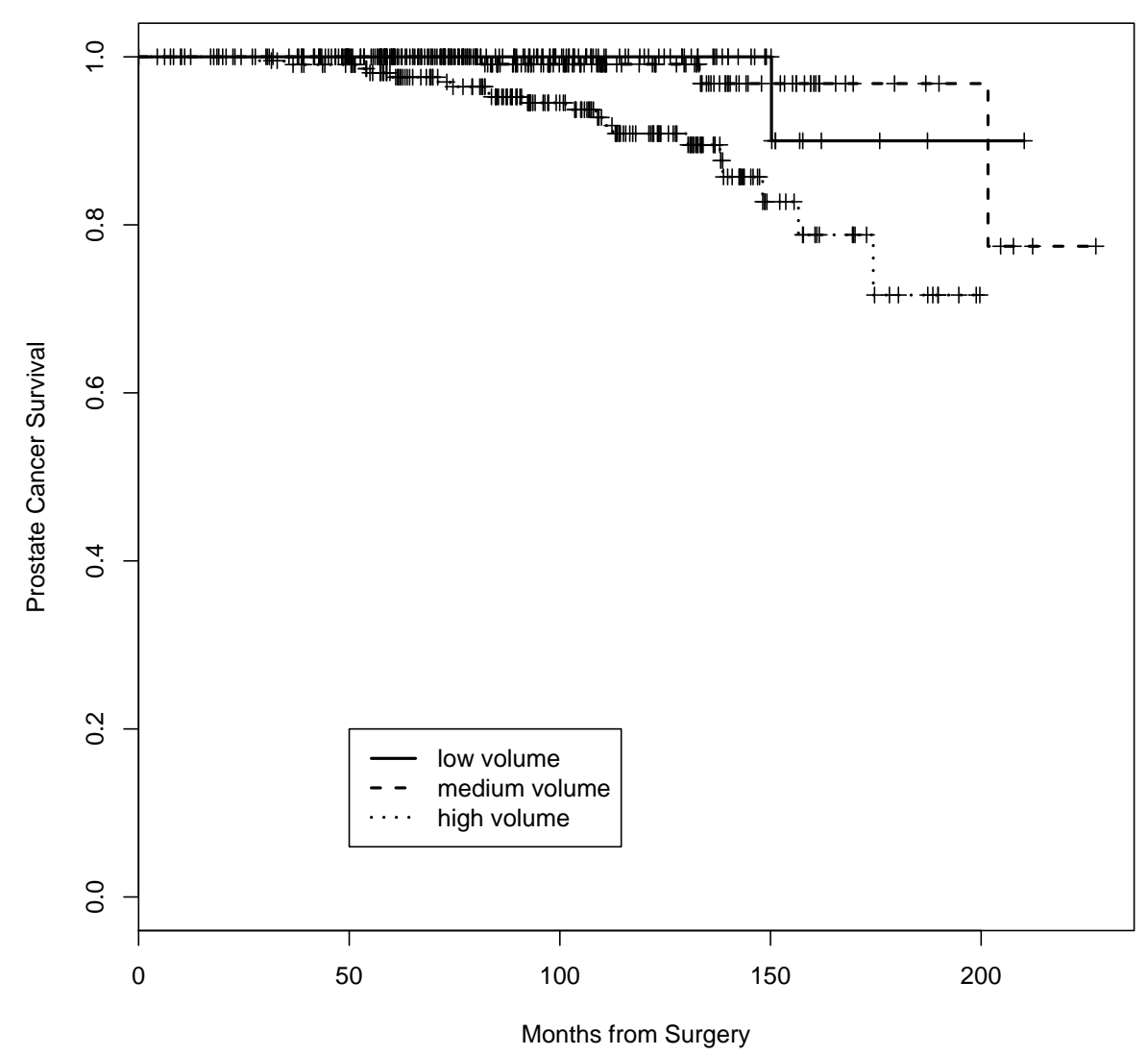

Fig. (3). Kaplan-Meier analysis representing time to prostate cancer specific death after surgery for tumor volume in all patients with Gleason grade $3+4$ and above. Tumor volume and censored values depicted as previously. Tumor volume was a significant predictor of prostate cancer specific death $(p=0.0003, \log$ rank test).

plying PSA velocity screening standards will lead to detection of high-risk tumors when they are low volume, or whether velocity-based screening methods are even practical, given the current confusion surrounding PSA testing in the medical community. Finally, altering existing screening procedures to detect cancers at smaller volumes carries with it considerable risks, since significantly more men with clinically indolent cancers will be discovered and treated unnecessarily.

There are several limitations to our study that deserve mention. The improvements in rates of recurrence and survival in low volume cancers might simply reflect lead-time bias. Were that true, we would expect to see a shift of time to recurrence to a later date in our small volume cancers, which we have not observed over the relatively long follow-up of this study. It is also possible that the low volume cancers are enriched for tumors of indolent phenotype. However, the greatest benefit of low tumor volume was observed in the cohort harboring the highest risk tumors - those with high PSAV and high percentage grade 4/5. Somewhat surprisingly, tumor volume had little influence on the incidence or timing of recurrence in the patients with low risk features (i.e. grade $4 / 5<5 \%$ and PSAV $<2$ ng.ml/yr) arguing against a lead-time bias or differences in phenotype, at least in this group. The differences in the effects of tumor volume on outcome between low and high risk cancers is not surprising in light of studies of watchful waiting compared to surgery where the greatest treatment benefits were observed in pa- tients in the high risk cohort [14]. Furthermore, in a randomized trial of watchful waiting and surgery in which most patients had moderate to high risk disease, surgical treatment was associated with an absolute risk reduction of death from any cause of 5\% and an absolute decrease in metastatic disease of $10.2 \%$, compared with 10 years of observation during which time tumor volume certainly increased significantly [15]. These studies suggest that increased volume in moderate and high risk cancers is associated with poorer survival.

Together, these observations strongly suggest that screening methods that increase detection and treatment of moderate and high risk tumors when they are at low volume will increase prostate cancer specific survival. However, the finding of little or no benefit in either disease recurrence or survival for low risk cancers argues strongly against indiscriminate detection and treatment of all prostate cancers. Clearly, better screening tools are needed to identify tumors that are potentially lethal. At the very least, better prognostic tools are needed for the tumors detected by current screening practices. What types of tools are likely to be helpful? Since Gleason score and tumor volume are the most powerful predictors of outcome in our series, imaging tools to estimate tumor size and Gleason grade in the entire tumor would be one possibility. However, size and grade do not capture the entire tumor phenotype, and it will be necessary to develop molecular biomarkers that predict outcome independent of grade, size and other clinical features. 


\section{CONCLUSIONS}

In moderate and high risk patients, tumor volume is highly associated with prostate cancer recurrence and prostate cancer specific death. Since the widespread application of prostate cancer screening has resulted in a decrease in tumor volume over time, one possible explanation of the decrease in prostate cancer deaths in North America is early detection of moderate and high risk tumors when they are more amenable to definitive local therapy.

\section{ACKNOWLEDGEMENTS}

John McNeal, MD performed prostate pathologic examination.

\section{ABBREVIATIONS}

$$
\begin{aligned}
\text { PSA, }= & \text { Prostate Specific Antigen } \\
\text { PSAV = } & \text { Prostate Specific Antigen Velocity } \\
\text { PCLO = } & \text { Prostate, Lung, Colorectal and Ovarian Cancer } \\
& \text { Screening Trial } \\
\text { ERSPC = } & \text { European Randomized Study of Screening for } \\
& \text { Prostate Cancer (ERSPC) trial } \\
\mathrm{CT}= & \text { Computerized tomography }
\end{aligned}
$$

\section{REFERENCES}

[1] Stamey TA, Caldwell M, McNeal JE, Nolley R, Hemenez M, Downs J. The prostate specific antigen era in the United States is over for prostate cancer: What happened in the last 20 years? J Urol 2004; 172: 1297-301.

[2] Andriole GL, Reding D, Hayes RB, Prorok PC, Gohagan JK. The prostate, lung, colon, and ovarian (PLCO) cancer screening trial: Status and promise. Urol Oncol 2004; 22(4): 358-61.

[3] Postma R, Schroder FH, van Leenders GJ, et al. Cancer detection and cancer characteristics in the European Randomized Study of Screening for Prostate Cancer (ERSPC)--Section Rotterdam. A comparison of two rounds of screening. Eur Urol 2007; 52(1): 8997.

[4] Henschke CI, Yankelevitz DF, Libby DM, Pasmantier MW, Smith JP, Miettinen OS. Survival of patients with stage I lung cancer detected on CT screening. N Engl J Med 2006; 355(17): 1763-71.

[5] Bach PB, Jett JR, Pastorino U, Tockman MS, Swensen SJ, Begg CB. Computed tomography screening and lung cancer outcomes. JAMA 2007; 297(9): 953-61.

[6] Stamey TA, McNeal JE, Yemoto CM, Sigal BM, Johnstone IM Biological determinants of cancer progression in men with prostate cancer. JAMA 1999; 281(15): 1395-400.

[7] Berger AP, Deibl M, Strasak A, et al. Relapse after radical prostatectomy correlates with preoperative PSA velocity and tumor volume: results from a screening population. Urology 2006; 68(5): 1067-71.

[8] D'Amico AV, Chen MH, Roehl KA, Catalona WJ. Preoperative PSA velocity and the risk of death from prostate cancer after radical prostatectomy. N Engl J Med 2004; 351(2): 125-35.

[9] Patel DA, Presti JC Jr, McNeal JE, Gill H, Brooks JD, King CR. Preoperative PSA velocity is an independent prognostic factor for relapse after radical prostatectomy. J Clin Oncol 2005; 23(25): 6157-62.

[10] Renshaw AA, Chang H, D'Amico AV. Estimation of tumor volume in radical prostatectomy specimens in routine clinical practice. Am J Clin Pathol 1997; 107(6): 704-8.

[11] Merrill MM, Lane BR, Reuther AM, Zhou M, Magi-Galluzzi C, Klein EA. Tumor volume does not predict for biochemical recurrence after radical prostatectomy in patients with surgical Gleason score 6 or less prostate cancer. Urology 2007; 70(2): 294 8.

[12] Thompson IM, Pauler DK, Goodman PJ, et al. Prevalence of prostate cancer among men with a prostate-specific antigen level < or $=4.0 \mathrm{ng}$ per milliliter. N Engl J Med 2004; 350(22): 2239-46.

[13] Carter HB, Ferrucci L, Kettermann A, et al. Detection of lifethreatening prostate cancer with prostate-specific antigen velocity during a window of curability. J Natl Cancer Inst 2006; 98(21): 1521-7.

[14] Lu-Yao GL, Yao SL. Population-based study of long-term survival in patients with clinically localised prostate cancer. Lancet 1997 349(9056): 906-10.

[15] Bill-Axelson A, Holmberg L, Ruutu M, et al. Radical prostatectomy versus watchful waiting in early prostate cancer. N Engl J Med 2005; 352(19): 1977-84.

(C) Brooks et al.; Licensee Bentham Open.

This is an open access article licensed under the terms of the Creative Commons Attribution Non-Commercial License (http://creativecommons.org/licenses/ by-nc/3.0/) which permits unrestricted, non-commercial use, distribution and reproduction in any medium, provided the work is properly cited. 